emptying disorders is the malfunctioning of the detrusor and/ or the sphincter muscles.

During the 34-year study period, 4965 paediatric urodynamic examinations were performed. 551 children had videourodynamic examinations, 210 had cystomanometry, and 4,215 had uroflow and ultrasound.

Videourodynamics showed normal functioning in 74 patients, vesicoureteral reflux in 301 children, and detrusor dysfunction with increased activity in 182 cases. Neurogenic bladder dysfunction was present in 51 patients, outflow disorder in 18 patients, and wide bladder neck in 23 patients.

Vaginal reflux was confirmed in 11 cases. Simple uroflow showed reduced urinary flow with residuum in 60, 43 without residuum, increased urinary flow in 1275 cases, intermittent urinary flow with residuum in 320 cases and regular urinary flow without residuum in 2517 cases.

Urodynamic examinations are needed to accurately diagnose abnormalities in the lower urinary tract

\section{A RARE FORM OF TUBERCULOSIS PRESENTATION}

Tudor-Constantin Pop*, Tiereanu loana. 'Maria Sklodowska Curie' Emergency Children's Hospital, Bucharest

\subsection{6/archdischild-2021-europaediatrics.365}

Case presentation: a 14 year-old female patient presents with fever (39.8 degrees Celsius), emesis and abdominal pain. Fever started four days earlier, was unremitting to antipyretics and prompted an initial ED visit after two days when leukocytosis with neutrophilia $(17.17 \times 103 / \mathrm{mm} 3$ and 14.31 x 103 respectively), elevated CRP (108.4 mg/L), minimal leukocyturia and hematuria $(25 / \mathrm{mm} 3$ and $10 / \mathrm{mm} 3$ respectively)were noted. A chest X-ray was performed showing left pachypleuritis. Oral Ciprofloxacin was prescribed and the patient was discharged. Symptoms persisted despite five doses of antibiotic prompting return to the ED after two days. She was admitted on suspicion of UTI.

Of note: 4 months prior she describes an episode of left inferior lobar pneumonia with pleural effusion that required drainage.

Clinical exam was relatively normal: no hemodynamic instability, dysuria or lumbar pain. New laboratory tests were ordered and revealed leukocytosis with neutrophilia (15.92 x $103 / \mathrm{mm} 3$ and $12.68 \times 103 / \mathrm{mm} 3$ respectively), rising CRP $(155.7 \mathrm{mg} / \mathrm{L})$, leukocyturia $(50 / \mathrm{mm} 3)$, proteinuria $(75 \mathrm{mg} / \mathrm{dL})$ and hematuria $(25 / \mathrm{mm} 3)$. Urine culture was negative. Renal ultrasound revealed slight right pyelectasia. Pyelonephritis with unkown agent was diagnosed and IV Ceftriaxone $70 \mathrm{mg} / \mathrm{kg}$ once daily was started.

During the next three days the patient had frequent fever episodes and continuously rising CRP (193 mg/L) and leukocytes $(19 \times 103 / \mathrm{mm} 3)$. Antibiotic therapy is changed to Meropenem $60 \mathrm{mg} / \mathrm{kg} / \mathrm{day}$ t.i.d. Fever continues and chest tightness is described. Further tests are ordered: blood cultures (negative), chest X-ray (left basal opacity interpreted as possible pleural effusion is noted), ESR (100 mm/h), fibrinogen $(625$ $\mathrm{mg} / \mathrm{dl}), \mathrm{C} 3, \mathrm{C} 4$, circulating immune complexes, rheumatoid factor, p-ANCA, c-ANCA, antinuclear antibodies, anti-dsDNA antibodies, QuantiFERON TB gold, HIV serology (negative) and a metabolic panel. Creatinine and urea are elevated (4.22 $\mathrm{mg} / \mathrm{dl}$ and $87 \mathrm{mg} / \mathrm{dl}$ respectively). Fractional excretion of sodium is $1.6 \%$ suggesting tubular damage. No sign of nephritic syndrome is noted and diuresis is normal. With supportive measures creatinine and urea values decrease $(2.72 \mathrm{mg} / \mathrm{dl}$ and $57 \mathrm{mg} / \mathrm{dl}$ respectively). Chest echography reveals a $21 \mathrm{~mm}$ thick pleural effusion. QuantiFERON TB gold test is positive prompting transfer to a specialized tuberculosis clinic. On patient follow-up kidney function returned to normal after specific TB reatment.

Renal TB is noted in literature, but the age and immune status of the patient, the clinical presentation, confounding laboratory findings and associated acute kidney injury with interstitial nephritis are of particular interst. Rare forms of renal TB (such as membranoproliferative glomerulonephritis) are noted, but usually in old and/or immunocompromised patients.

\section{AUDIT OF CURRENT UTILISATION OF PAEDIATRIC INDIRECT MAG3 RADIONUCLIDE CYSTOGRAPHY IN CHILDREN'S HEALTH IRELAND}

Caoimhe Howard, Cormac Duff*, David Curran, C Brenner, I Robinson, Salvatore Cascio, Maria Stack. Children's Health Ireland at Crumlin and Temple Street, Dublin, Ireland

\subsection{6/archdischild-2021-europaediatrics.366}

To assess clinical characteristics and indication for imaging of the patients who have had indirect MAG3 cystography (IRC) performed in CHI at Crumlin and Temple Street, and whether it changed patient management.

In this retrospective audit we identified all children who had IRC performed in Temple Street and Crumlin in the last 4 years by searching the radiology systems. Data collected included age of patient at time of scan, indication for scan, results of scan, whether a conventional micturating cystourethrogram (MCUG) had previously/subsequently been performed, and what changes were made to management.

$\mathrm{N}=36$ patients were identified ( 3 male). Mean age at scanning was 9 years 5 months. The most common indication was recurrent UTI (33/36), with additional renal scarring in $14 / 33$ of these. 12 patients had had a previous MCUG, and 11 of these demonstrated reflux. 5 patients had previously had a STING procedure and one had ongoing reflux on IRC and was referred for surgery. 8 patients had reflux demonstrated on IRC. 4 of these went on to have surgical intervention based on their IRC - 1 had a ureteric reimplantation, and 3 had STING procedures. 3 patients had a standard MCUG following a negative IRC due to high suspicion of reflux. One of these displayed grade 1 reflux, one displayed grade 2 bilateral reflux, and one was normal. The patient with grade 2 bilateral reflux also had renal scarring and went on to have a STING procedure performed.

IRC is a safe, non-invasive alternative to MCUG in older children with suspicion of reflux. Demonstration of reflux on IRC can be helpful in decision making regarding further intervention for patients with recurrent UTIs, particularly with renal scarring. For the majority of patients a negative result can reassuring, without the patient having an invasive procedure. 